\title{
Conflictos y negociaciones en la construcción metropolitana. El corredor Oeste del Gran Buenos Aires (1854-1950)
}

Melisa Pesoa. Universitat Politècnica de Catalunya, Barcelona, España. Ana Gómez-Pintus. Conicet, La Plata, Argentina.

RESUMEN | El presente texto pretende indagar, en la larga duración, sobre el elenco de actores que intervienen en los procesos de producción del suelo urbano de la expansión de la ciudad de Buenos Aires y los cambios institucionales que se producen en las decisiones acerca de cómo debe ser la ciudad. A partir del análisis del eje Oeste de crecimiento de la ciudad en el periodo 1854-1950, desarrollamos una aproximación que pone en juego la combinación de directrices "desde arriba" acerca de cómo debe ser la ciudad (normativas, reparticiones técnicas), con los requerimientos "desde abajo" de los agentes locales. El estudio pone en evidencia que, mientras en el siglo xix los conflictos y negociaciones para el ajuste de las normativas a su materialización en el territorio se dirime entre los "vecinos", hacia finales de siglo, con una nueva organización institucional, comienza una creciente burocratización para consolidar los nuevos crecimientos, la cual resta protagonismo a los actores locales.

PALABRAS CLAVE | áreas metropolitanas, expansión urbana, historia urbana.

ABSTRACT In this article, we explore the variety of actors involved in the process of production of urban land and the institutional changes produced by the decisions about how the city had to be. With the analysis of the urban growth along Buenos Aires' west axis for the period 1854-1950, we develop an approximation that shows the combination of "top-down" guidelines about how the city must look like (regulations, the role of technical offices) with "bottom-up" requirements from local agents. This reveals that, while in 19th century, the conflicts and negotiations for adjusting the regulations to the actual materialization of the city were solved among the neighbors, towards the end of the century, with a new institutional organization, an increasing bureaucratization in the consolidation of the new urban growth began, which diminished notoriety to local actors.

KEYWORDS | metropolitan areas, urban sprawl, urban history. 


\section{Introducción}

La llegada del ferrocarril a Buenos Aires en el ańo 1854 cambia para siempre la relación entre la ciudad y su hinterland. Los que hasta el momento habían sido pequeños poblados surgidos a la vera de los caminos, comienzan a integrarse lentamente a la ciudad. Primero lo hacen como partes discontinuas vinculadas por la infraestructura ferroviaria; pero, ya en el siglo $\mathrm{xx}$, se inicia el completamiento de los intersticios entre los poblados. Poco a poco, la urbanización se extiende en un continuo edificado más allá de los propios límites de la ciudad, apoyada en cambios económicos y culturales -cambios en la familia tradicional, nuevas actitudes hacia el ocio y el tiempo libre-, en el avance de las infraestructuras -primero ferroviarias y luego viales-, en la popularización del transporte público automotor y el uso creciente del automóvil privado.

A lo largo de todo este proceso, se reconoce que los antiguos pueblos se transforman y van quedando incluidos en el continuo suburbano (figura 1). Y si antes primaba el interés sobre la tierra productiva, ahora el suelo urbano será el objeto de interés, sobre el cual se producirá un proceso especulativo que podemos observar por primera vez en el área de influencia de Buenos Aires. ${ }^{1}$

Nos interesa, entonces, explorar este ámbito en el momento en que confluyen procesos de producción del suelo urbano desde la lógica ejidal, hasta los procesos modernos de suburbanización. Para ello, pondremos el foco en los encuentros entre las directrices e intervenciones que vienen "desde arriba" -es decir, la acción del Estado (nacional, provincial o municipal) a través de sus oficinas técnicas, leyes y reglamentos- con las intervenciones surgidas "desde abajo" - producto de las problemáticas y actores locales-. Entendemos, así, la ciudad como una arena de conflictos y negociaciones.

En la colisión que se produce entre una forma institucional y normativa de producir la ciudad, y las realidades y poderes locales, nos enfocamos en los agentes que intervienen en la producción del suelo urbano y el contacto entre ellos. En esta línea se destacan los aportes iniciales de Oszlak (1982), quien a partir de la década de 1850 propone la construcción progresiva de un orden estatal, el cual da lugar a una nueva frontera entre el dominio legítimo del Estado y de la sociedad. Como sabemos, esta frontera nunca fue rígida, sino, al contrario, irregular y porosa. Esto nos lleva a plantear también la perspectiva de la organización dinámica del Estado y sus agencias (Bohoslavsky \& Soprano, 2010). Abordar el Estado como actor en sus diferentes escalas y niveles de acción nos ayuda a comprender las implicancias que las políticas públicas y las acciones legislativas pudieran tener sobre los procesos de urbanización y ordenamiento territorial.

El análisis nos permite poner en crisis la cuestión de "la ausencia del Estado" -una idea constantemente reiterada, particularmente desde los ámbitos no

$1 \longdiv { \text { Alrededor de 1860, junto con la consolidación del Estado nacional, la ciudad de Buenos Aires } }$ asume un doble rol, como capital de Argentina (albergando las institucionales del Estado nacional) y como capital para la provincia del mismo nombre. A partir de 1880 se produce un cambio administrativo que mantiene a la ciudad de Buenos Aires como capital nacional, en tanto se funda una nueva ciudad, La Plata, como sede del estado provincial. 
académicos- en la construcción urbana fuera de la ciudad de Buenos Aires. Muy al contrario, la existencia de diferentes oficinas técnicas (como el Departamento Topográfico y, más tarde, el Departamento de Ingenieros y el Ministerio de Obras Públicas), junto con las redes de poder local (como las Comisiones de Solares o las Comisiones Municipales y, por supuesto, los propietarios de las tierras), configuran un elenco de actores que puede dar cuenta de la relación entre el Estado y la sociedad en diferentes etapas.

Por otra parte, es fundamental plantear esta investigación en diálogo con aquellas que abordan el funcionamiento del mercado de tierras y sus efectos sobre la configuración del espacio urbano. En principio, cabe mencionar el trabajo de Topalov (1987), sobre el mercado de la vivienda en Francia; y en el contexto latinoamericano, la investigación de Schteingart (1989), que comparte, junto al trabajo de Jaramillo (1981), el interés por reconocer el rol de los productores de vivienda en el proceso de expansión. Asimismo, nos referimos a los trabajos de Clichevsky $(1975,2002)$ sobre el mercado de tierras, o los de Smolka $(1981,1987)$, acerca de la formación de los precios de tierra, e incluso los más recientes de Lovera (2012) o Sabatini y Arenas (2000), que renuevan estas discusiones.

Finalmente, este trabajo también se ancla en el más amplio grupo de autores que estudian pasado y presente del Gran Buenos Aires. Entre ellos se reconocen los más recientes: Favelukes, Novick y Zanzottera (2016), quienes analizan las cartografías metropolitanas; el ensayo de Gorelik (2015), que reivindica la comprensión del Gran Buenos Aires como tal; o el reciente número de la revista Anales del IAA, dedicado a los Bordes Metropolitanos (2017), con artículos que, desde diferentes perspectivas y temporalidades, retoman las áreas de borde y periferias como objeto de estudio.

Sobre estas líneas de trabajo, pretendemos explorar la forma en que se concretan estas negociaciones entre actores en la construcción material de la ciudad. Planteamos que, pese a que la existencia de una normativa y la presencia de cuerpos técnicos encargados de realizar o verificar los trazados propuestos nos pueden llevar a suponer un modelo de ciudad impuesto sobre el territorio, la construcción de la misma se da en el marco de una relación compleja entre los actores que intervienen en dicho proceso. Por ello proponemos, como hipótesis de trabajo, que las posiciones que adoptan los diferentes actores, ya sean privados o estatales, a lo largo del periodo de estudio, se vinculan a mayor escala con el proceso de fundación del Estado argentino. Este se conforma mediante un proceso lento de institucionalización y centralización que se traduce en un desplazamiento de las capacidades de los poderes locales en favor del incremento de las capacidades del nuevo Estado provincial, un proceso no exento de constantes debates y pujas registradas entre la segunda mitad del siglo xix y las primeras décadas del xx.

En tanto resulta posible, hemos evitado mirar este fenómeno desde la gran ciudad, Buenos Aires; nos enfocamos, más bien, en examinar los problemas desde la realidad de los pequeños poblados y su crecimiento, atendiendo a sus propias lógicas. Esto nos permite explorar la escala local de las decisiones urbanas que van conformando los diferentes fragmentos que más tarde serán el continuo edificado de la ciudad de Buenos Aires. 

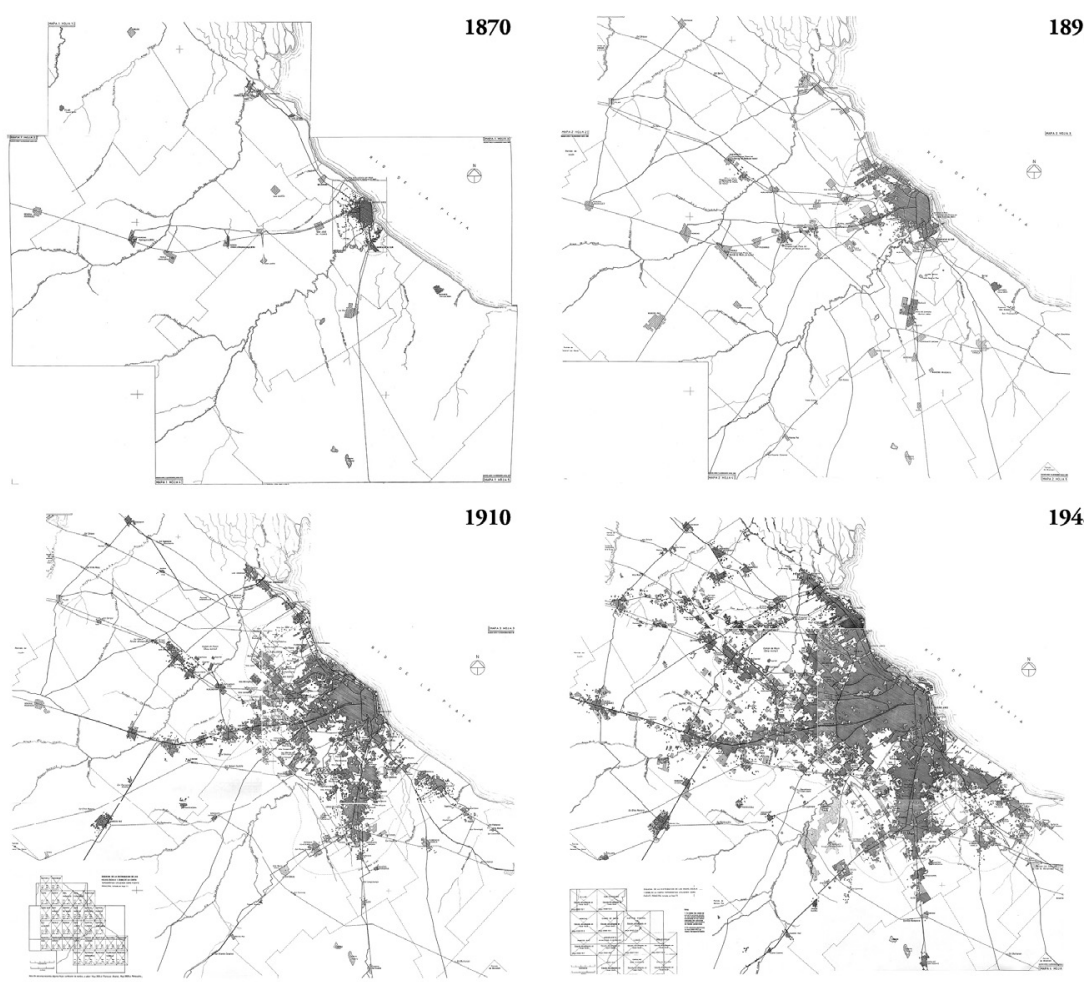

Figura I | Crecimiento de Buenos Aires, 1870, 1895, 1910 y 1948.

Los mapas de Vapńarsky son producto de una extensa investigación y presentan una síntesis de las manzanas edificadas. Pese a no reflejar el crecimiento poblacional, aportan datos fieles sobre la ocupación real en diferentes momentos de la expansión (Favelukes, Novick, \& Zanzottera, 2016)

FUENTE: VAPÑARSKY, 2000

De esta manera, los principales aportes de esta investigación se sintetizan en tres ejes. El primero es una exploración de la larga duración sobre la construcción de la ciudad, destacando el pasaje desde una lógica ligada a lo ejidal, a una metropolitana. El segundo tiene que ver con el estudio de las relaciones y tensiones entre los actores que producen la ciudad, una mirada poco desarrollada hasta ahora en el ámbito de estudio. El tercero pretende destacar una mirada de la construcción de la ciudad desde su periferia y no desde el centro.

Este análisis se lleva a cabo desde una metodología empírica. Se propone como recorte geográfico el eje Oeste de expansión de Buenos Aires, por ser el eje de crecimiento más antiguo hacia el interior, vinculado al Camino Real que llevaba a Potosí, y porque fue el primero sobre el cual se implantó el ferrocarril. Hemos tomado el eje desde el centro de la ciudad hasta Moreno (unos $40 \mathrm{~km}$ de largo), dado que es el tramo que -de acuerdo con lo analizado hasta el momento- registra mayor cantidad 
y diversidad de situaciones. Asimismo, esperamos que nos sirva de base para luego efectuar comparaciones con los otros ejes de crecimiento.

A lo largo del proceso de consolidación del eje Oeste, hemos identificado dos etapas factibles de analizar. La primera arranca en 1854, cuando suceden dos hechos centrales en el área de estudio: por un lado, se promueve la Ley de Municipios; y por otro, se establece la primera línea ferroviaria entre Buenos Aires y el pueblo de San José de Flores. Esta etapa finalizaría en 1913, año que utilizamos para poner en valor nuevamente dos situaciones: una es la promulgación de la ley de "Fundación de pueblos y ampliación de ejidos", y la otra es la construcción del primer barrio parque en el área de influencia de Buenos Aires, una tipología residencial que dará lugar a la consolidación del crecimiento intersticial entre los antiguos pueblos que conformaban el eje. La segunda etapa arranca desde este punto -cuando se inicia una tendencia a la venta de lotes destinados a barrios parque o viviendas de segunda residencia-, hasta comienzos de los años cincuenta, cuando las lógicas de expansión dan otro giro hacia nuevos patrones.

El trabajo se apoya en fuentes primarias y, muy especialmente, en fuentes cartográficas relacionadas con el catastro, lo cual, a su vez, nos permite poner en relieve la labor técnica realizada por agrimensores e ingenieros durante los siglos XIX y XX. Para el primer periodo, se han estudiado los Registros Gráficos de las Propiedades Rurales de 1830, 1864 y 1890, además de los planos por partido construidos para este último ańo. Asimismo, se han analizado las mensuras de los pueblos investigados, algunos de cuyos planos reproducimos en este artículo. Para el segundo periodo, se han estudiado los planos por partido realizados por el Ministerio de Obras Públicas de la Provincia, así como los planos de los diferentes loteos que aparecen en nuestra área de estudio. El estudio de las fuentes cartográficas reviste un singular valor para el estudio de la transformación del suelo rural en urbano.

El presente texto se divide en tres partes. En la primera explicamos el origen de los núcleos que conforman el eje Oeste y cómo era la situación en el área antes de la llegada del ferrocarril. Los otros dos apartados coinciden con los dos periodos identificados. Para cada uno de ellos reconocemos instancias de conflicto entre las leyes, modelos vigentes y las prácticas llevadas a cabo para adaptarlos a la realidad local. Finalmente, señalamos algunas conclusiones de este estudio y algunos puntos de discusión.

\section{El eje Oeste: los “pueblos” pioneros}

En 1824, por iniciativa de Bernardino Rivadavia, se creó el Departamento Topográfico de Buenos Aires (entonces llamado Comisión Topográfica; en adelante, Dт). ${ }^{2}$ Este cuerpo técnico fue el encargado de, entre otras incumbencias, conformar

2 A pesar de una baja en la actividad durante la etapa rosista, el DT tuvo continuidad hasta 1875 , cuando quedó englobado dentro del recién creado Departamento de Ingenieros, que contaba con cuatro secciones: Obras Públicas, Geodesia, Puentes y Caminos, y Trabajos Catastrales. Más tarde, en el ańo 1885, se creó el Ministerio de Obras Públicas (hasta ese momento la Provincia tenía solo dos ministerios: Gobierno y Hacienda), que englobaba al Departamento de Ingenieros, además de otras dependencias, como el Departamento de Higiene (hoy Ministerio de Salud), el Museo, la Biblioteca, el Observatorio, la Escuela Santa Catalina y la Escuela de Artes y Oficios. 
el mapa de las propiedades de la provincia de Buenos Aires. Pese a que inicialmente estaba enfocado en la propiedad rural, fue el encargado de trazar los pueblos y ciudades del interior bonaerense. La normativa elaborada desde la década de 1810 propuso un modo de hacer ciudad que podemos denominar "modelo republicano". ${ }^{3}$ Se trata de una ciudad en cuadrícula, con una plaza central y una zona productiva alrededor (el ejido). Pese a algunas semejanzas formales con la ciudad colonial, se trata de un producto auténticamente republicano, propio del siglo XIX, con raíces en la época borbónica. Sobre este modelo se establecerán más de cien nuevas poblaciones en toda la provincia de Buenos Aires, principalmente en la segunda mitad del siglo XIX (Pesoa, 2016).

El procedimiento era casi siempre el mismo, aunque con particularidades para cada situación. Normalmente se partía de la iniciativa de algunos propietarios locales que pedían autorización al gobierno para establecer un pueblo. Una vez aprobada la fundación, los propietarios cedían terreno para el mismo. El trazado y la parcelación iban a cargo del agrimensor, pagado por los interesados. El plano producido por este era finalmente aprobado por el DT para su posterior concreción sobre el terreno. La adjudicación de los solares era competencia de la Comisión de Solares -más tarde Comisión Municipal-, una figura creada en la década de 1820 para gestionar las tierras del ejido (volveremos sobre este punto en el primer periodo). Como es natural, los solares alrededor de la plaza solían acabar en manos de los miembros fundadores del pueblo, siendo que, además, se prescribían algunas normas edificatorias para estas parcelas que los pobladores de bajos recursos no estaban en condiciones de afrontar.

Sin embargo, no todas las poblaciones siguieron este modelo fielmente. Existían poblaciones antiguas, sobre todo del siglo XviII, originadas en postas, alrededor de capillas o en puestos de vigilancia fronteriza, que tienen formas más arcaicas. Los pueblos ubicados a lo largo del eje Oeste que estudiamos pertenecen a esta categoría: Morón y Merlo eran caseríos formados en torno a una capilla que funcionaba como lugar de paso en el Camino Real.

Tal como relata Birocco (2009), Morón surgió en 1769 a raíz de la donación que hizo Marcos de Alarcón, propietario de las tierras, para la construcción de una capilla. Casi una década después, los herederos de Alarcón vendieron los primeros solares a quienes serían los pobladores pioneros. Durante las primeras décadas del siglo XIx, el caserío ocupaba unas diez cuadras alrededor de la plaza y la capilla, pero carecía absolutamente de planificación, lo que generaba múltiples discusiones entre los vecinos por los accesos públicos y el área de expansión.

Las reglamentaciones a las que hacemos referencia son las siguientes: disposiciones generales sobre la fundación de pueblos de 15 de septiembre de 1814; reglamentación sobre edificios y calles de pueblos del 14 de diciembre de 1821; decreto sobre demarcación de los pueblos de campaña, 16 de abril de 1823; decreto designando en los fuertes de la nueva línea de frontera, el terreno para las poblaciones y tierras de pan llevar, 28 de abril de 1828; la ley de 24 de enero de 1854; la Ley 695 de 31 de octubre de 1870, llamada Ley de Ejidos; la Ley 817 de inmigración y colonización, sancionada el 19 de octubre de 1876, también llamada Ley Avellaneda; la ley de 26 de agosto de 1910; y, por último, la Ley 3487 del 17 de junio de 1913. 
El poblado de Merlo, por su parte, ubicado a 300 varas del Camino Real, surgió en torno a una capilla establecida por Francisco de Merlo, el propietario de las tierras. Este cedió tierras a algunos pobladores que conformaron el núcleo inicial del pueblo y consiguió su aprobación tras sucesivas solicitudes que hizo al Cabildo -la primera en 1738- (Canedo, 2013). La real cédula de fundación llegó recién en 1754 y la autorización definitiva en 1755 .

Estos poblados eran caseríos en medio de una gran área dedicada a la agricultura (dado que, según la legislación colonial, debían ser tierras "de pan llevar” y, en menor medida, dedicadas a la cría de animales). En estas poblaciones se localizaban los trabajadores manuales, tales como los zapateros, carniceros, panaderos, entre otros, y los comerciantes. Durante el primer cuarto del siglo xix, la actividad comercial se expandió notoriamente, llegándose a contabilizar 41 comerciantes en 1825 en Morón (Sáez, 2014). En el caso de Merlo, más alejado de Buenos Aires, el censo de 1838 indica que había en el pueblo 94 personas viviendo en 15 hogares, mientras que alrededor predominaba la cría de ganado, pese a estar prohibida, por ser tierra de pan llevar.

Como veremos a continuación, estos antiguos poblados se "refundan" con la llegada del ferrocarril. Por lo tanto, se manda "arreglar" el pueblo de acuerdo con principios regulares, intentando adaptar el caserío al modelo utilizado por los técnicos del DT. Aunque los resultados no se parecen a los pueblos localizados en medio de la Provincia y alejados de la ciudad, permanecen los elementos básicos: el trazado reticular y la plaza principal.

Por lo tanto, hasta aquí destacamos, por un lado, una ocupación organizada por los pobladores locales, alentada y avalada por el propietario de las tierras; y, por otro, una edificación de la ciudad con una "planificación" mínima basada en la sola consigna de respetar la plaza (figura 2).

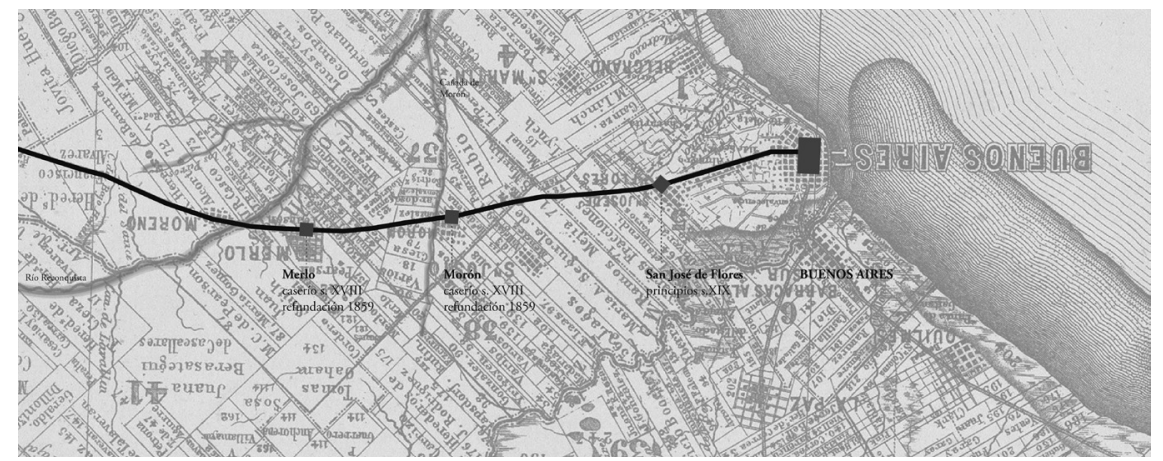

FIGURA 2 | Diagrama de los poblados establecidos antes de 1854 sobre el Camino Real del Oeste

FUENTE: ELABORACIÓN PROPIA CON BASE EN REGISTRO GRÁFICO DE TIERRAS DE I 864 


\section{Periodo 1: La fiebre ferroviaria}

El Ferrocarril Oeste (FCO) fue la primera línea ferroviaria del país. Comenzó siendo una empresa privada, una sociedad anónima organizada por un grupo de promotores que actuó entre 1854 y 1862. Luego fue comprada por el Estado provincial y, en 1889, vendida a capital inglés (Schwarzer \& Gómez, 2006). La intención inicial de esta línea era servir para el transporte de mercancías de exportación e importación, pero finalmente el transporte de pasajeros resultó un negocio imposible de eludir, por las amplias ganancias que dejaba.

Uno de los negocios implícitos en el ferrocarril era el beneficio del valor de la tierra, pues la vía revalorizaba el suelo adyacente por incremento de las operaciones de compraventa. Evidentemente, el valor de la tierra sería mayor si la tierra ya formaba parte de un pueblo que cuando era tierra rural, por lo que la mayor especulación se producía alrededor de las estaciones próximas a poblados existentes. Así sucedió en Morón, donde la solución fue la siguiente: si las vías pasaban por terrenos de propiedad pública, estos se cedían a la empresa, mientras que, si los terrenos eran de propiedad privada, se expropiaban. De esta manera, los propietarios se beneficiaban junto con la empresa, y había una gran expectativa por la ubicación de las estaciones. En Morón, un área equivalente a 30 manzanas compradas por 16.000 pesos en 1855 , se vendía a 100.000 pesos la manzana tras la llegada del ferrocarril (Sargent, 1974). ${ }^{4}$ Cortés Conde (1979, pp. 164-166) estima que, entre 1894 y 1904, los precios del suelo en la Provincia se multiplicaron por tres, especialmente en los sitios por donde pasaban nuevas infraestructuras.

En febrero de 1859 el tren llegó a Morón, que se convirtió en un centro de recreo próximo a la ciudad de Buenos Aires. En abril de 1860 arribó a Moreno, que se consolidó como cabecera de línea por un tiempo. Así, la llegada del ferrocarril suponía la "refundación" de los pueblos, y renacía, en consecuencia, la preocupación por el trazado de sus manzanas y calles. Volvamos al caso de Morón para verificar cómo se produjo la ciudad de una forma muy diferente a partir de la llegada de la infraestructura ferroviaria, donde los propietarios llevaban unos ańos especulando con su arribo.

Cuando llegó el ferrocarril en 1859, los propietarios que tenían tierras frente a las vías se negaron a la propuesta de la Municipalidad de ceder una parte de terreno para ensanchar las calles. Por ello, y para regularizar la traza, se pidió la intervención

El relato de Mulhal (1875 [1869], pp. 124-125) puede ayudarnos a visualizar el estado en que se encontraban los pueblos de la zona en la primera mitad de la década de 1860 , una vez instalado el ferrocarril. Según el autor, en Morón el precio de la tierra rural varía entre $\$ 1.000$ y $\$ 6.000$ pesos por cuadra, mientras que las parcelas urbanas tienen unas 10 x 50 varas y se venden a partir de 10.000 pesos. El autor comenta, además, que las calles están bien trazadas, la plaza plantada y los edificios públicos y escuelas son amplios. En el caso de Merlo, Mulhal indica que el pueblo tiene 456 habitantes, una capilla gótica y escuelas a las que asisten 174 niños; observa también que el valor de las parcelas se multiplicó por 20 en los últimos cinco años. En Moreno, había unas 50 casas, donde vivían 372 personas (de un total de 2.329 que tenía todo el partido) y una nueva iglesia, pero llama la atención sobre el declive del pueblo desde que dejó de ser el final de línea del fco. 
del Departamento Topográfico, ${ }^{5}$ que envió al ingeniero Pedro Benoit a efectuar la traza del pueblo y arreglar las irregularidades producto de una ocupación sin control.

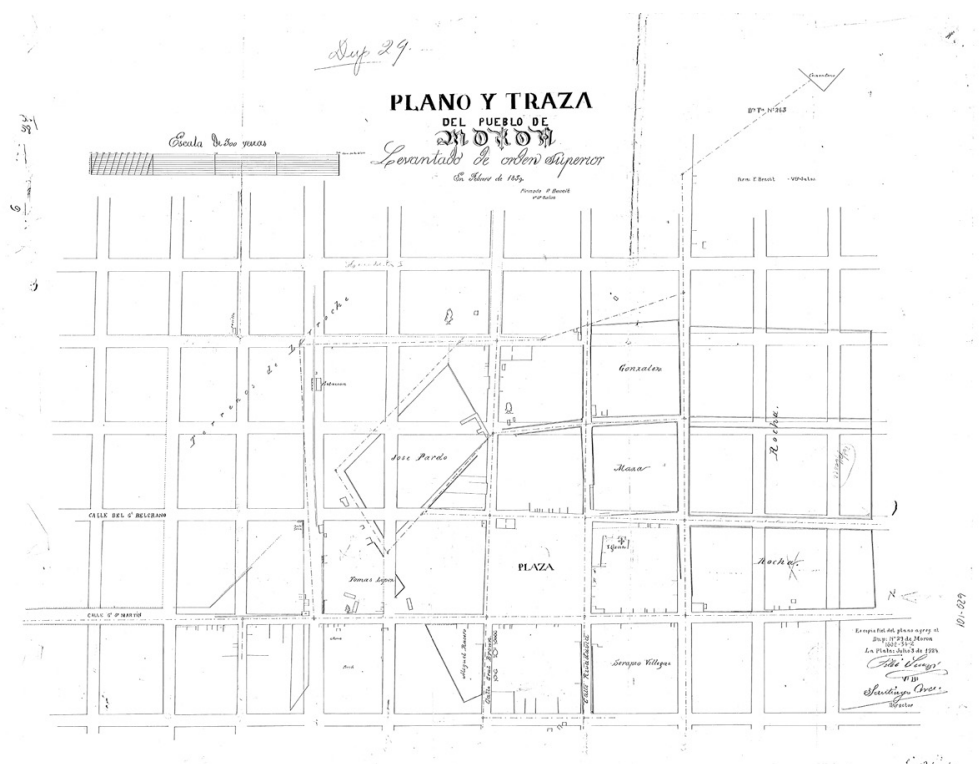

Figura 3 | Plano del pueblo de Morón en 1859, realizado por Pedro Benoit

FUENTE: DEPARTAMENTO DE INVESTIGACIÓN HisTÓRICA Y CARTOGRÁfiCA (DIHC), DiRECCiÓN DE GeOdeSia, MiNisTerio DE INFRAESTRUCTURA, PROVINCIA DE BUENOS AIRES

En su diseño (figura 3), Benoit mantuvo el centro del pueblo en la plaza y el ferrocarril quedó en un segundo plano; no obstante, se resolvió dar el ancho de 30 varas $(26 \mathrm{~m})$ a la calle de la vía, porque la Municipalidad así lo pidió. ${ }^{6}$ Para el resto de las calles, Benoit intentó mantener la forma anterior, realineando las casas. El único conflicto se presentó con el terreno del Señor Pardo:

En las calles en que no ha sido obstáculo se les ha dado el ancho de 20 varas [lo usual eran 16 varas], por acuerdo de la Municipalidad; quedando conforme los vecinos a excepción del señor Pardo, que no se ha conformado absolutamente con esta operación. En la quinta de este señor, que quedaría siempre mal situada con cualquier proyecto de traza que se adoptase, he trazado tres calles, habiendo mandado a abrir los señores municipales, dos de ellas que toman parte de la quinta del señor Don Tomás López, quien ha quedado muy conforme a pesar de llevarle esta calle gran cantidad

5 En el caso de Morón, el FCo procede de una manera diferente a como lo había hecho en otros pueblos, como San José de Flores. En Morón pasaba la vía por el centro de la calle de 20 varas (17,32 m), ocupando 16 varas, para evitarse expropiar, mientras que, en Flores, había pasado la vía por el centro de una manzana comprada a sus dueños.

6 Es preciso recordar que no había normativas ni antecedentes sobre la inclusión del ferrocarril en la trama urbana. La única referencia era el tramo que se había realizado en la Capital, y es la que reproduce Benoit para la traza de Morón: combinar la calle con las vías en un paseo arbolado, que por esta razón debe ser más ancho que una avenida al uso. 
de árboles frutales. (Mensura no 2 del partido de Morón, 1859, Departamento de Investigación Histórica y Cartográfica [DIHC]. Destacados nuestros)

Vamos a detenernos en dos aspectos importantes que se desprenden del análisis de la mensura. En primer lugar, la misma indica que el trazado fue acordado con la Municipalidad y con los vecinos (a excepción de uno de ellos). Sin embargo, en el caso de este vecino, aparecen alternativas de negociación: cesión de una de las calles o indemnización por parte de la Municipalidad en cuanto hubiera dinero disponible.

Estos hechos nos demuestran la preocupación del DT y sus técnicos por arribar a una traza acordada por todos los propietarios involucrados, en lugar de imponer un trazado "desde arriba". De esta manera, el agrimensor asignado actuaba como mediador entre un modelo de ciudad "ideal" (de acuerdo a la norma) y los intereses locales, donde las instrucciones recibidas por parte del DT podían ser objeto de múltiples adaptaciones para casar con los intereses de los agentes locales.

Este es un hecho de gran relevancia, dado que nos revela una instancia de consenso que subraya una hipótesis ya planteada por Garavaglia y Gautreau (2011, pp. 81-82) para la mensura de propiedades rurales en la campańa bonaerense durante el siglo XIx. Esto es, que no se buscaba tanto una exactitud técnica de la mensura, como la aceptación del trabajo del agrimensor por parte de los propietarios de los terrenos involucrados en la medición. Es decir, que se trataba de un procedimiento consensuado basado en la comprobación de títulos de propiedad y "hechos existentes" con el fin de evitar futuros pleitos.

Por lo tanto, si bien la existencia de normativa sobre la forma de la ciudad nos puede llevar a pensar en un modo "estandarizado" de producir pueblos, la realidad demuestra que el trazado debía adaptarse a situaciones diversas, tanto de índole natural (como la topografía del terreno) y artificial (límites de la propiedad por urbanizar, presencia del ferrocarril, etcétera), como también social (existencia de antiguas edificaciones, respeto de las alineaciones más antiguas o de los lugares de referencia del poblado).

Y es que el cuerpo normativo no solo regulaba la forma que debía tener la ciudad, sino que también determinaba la existencia de un agente clave para la producción de la misma: la Comisión de Solares, más tarde denominada Comisión Municipal. Las Comisiones de Solares se crearon en 1825 (Decreto 735 del 19 de enero de 1825), como parte de las iniciativas emprendidas durante el periodo rivadaviano para mejorar la administración del territorio, aunque la idea ya estaba presente desde 1814. ${ }^{7}$ Las comentamos, sin embargo, en este segundo periodo, pues su actuación en el área de estudio fue más relevante durante estos años.

El objetivo de las Comisiones de Solares era articular las decisiones de carácter local con las centrales en cuanto a tierras públicas y particulares, debido a lo cual trabajaron en estrecha colaboración con el Departamento Topográfico en la gestión

$7 \quad$ Ley de 15 de septiembre de 1814 (basada en una anterior de 9 de agosto de 1824): la ley especifica que se formará una Comisión compuesta por el alcalde, el cura y dos vecinos hacendados del partido, encargada de "allanar las dificultades" en el trazado y adjudicación de terrenos. Se encargará de facilitar los gastos para la mensura del pueblo, proponer mejoras, Reglamento de Policía y del repartimiento de suertes, en trato directo con el gobernador a través del Ministerio de Gobierno. 
de la adjudicación de lotes urbanos y ejidales. La ley de 1825 estableció una Comisión -formada por el juez de paz y dos vecinos- encargada de examinar los derechos de propiedad de los solares y, en el caso de los terrenos desocupados, repartirlos de manera gratuita a los individuos que desearan poblarlos (priorizando, de existir, los títulos de propiedad), observando ciertos requisitos de edificación, materialidad y cercos.

Las Comisiones de Solares tuvieron continuidad durante el periodo rosista y se renovaron con la promulgación de la Ley de Municipalidades de 1854 -recordemos que la primera Constitución provincial (1854) delegó en la Ley 35 (1854) la organización municipal-, porque sus funciones y deberes pasaron a las municipalidades de campaña (Cricelli \& Galcerán, 2013). A partir de ese momento se denominaron Comisiones Municipales y fueron las que, como en el caso de Morón que hemos relatado, intervinieron, junto con el agrimensor, para consensuar la traza definitiva del pueblo.

En el proceso de reordenación de los pueblos de campaña tras la reorganización del Departamento Topográfico, el Gobierno ordenó la formación de Comisiones en cada pueblo. Cada una de ellas debía estar compuesta de tres miembros, escogidos por el juez de paz. Por lo tanto, hay que tener en cuenta que los que las conformaban eran miembros de las grandes familias del área, por lo que tenían un cierto poder a nivel local. Su actuación fue variable: muchas veces estas Comisiones ayudaron en el trazado del pueblo y otras veces no estaban formadas cuando llegaba el agrimensor a efectuar sus tareas.

El rol de la Comisiones a nivel local debería ser entendido en el contexto de los cuestionamientos surgidos a partir de mediados del siglo XIX acerca de cómo organizar la campańa y la urgencia de definir las municipalidades. Hasta el momento, los diferentes partidos de la campaña se encontraban bajo el control del jefe de policía y juez de paz -instituciones surgidas como sustitutos de emergencia tras la extinción de los Cabildos en 1821-, en un contexto en que la campaña bonaerense cobraba mayor vida política. La pregunta en aquel momento era cuánta autonomía debía darse a los municipios, entendiendo que se estaba frente a una situación muy diversa que buscaba ser resuelta con una fórmula general (Heras, 1949).

La existencia de las Comisiones nos permite destacar su rol de mediador entre el poder del Estado provincial y las autoridades locales, tal como han hecho otros investigadores para otras zonas del interior de la Provincia (Aliata \& Loyola, 2005; Canedo, 2011, 2013, 2014; D’Agostino, 2015). Sin embargo, durante las últimas décadas del siglo XIX, en general el rol de la Comisión Municipal en la adjudicación fue decreciendo debido al surgimiento de un mercado de suelo urbano que ya no requería de un fomento del poblamiento, por lo que se centró en la provisión de servicios, equipamientos e infraestructura.

La costura que supone el ferrocarril, y que les faltaba a los poblados del periodo anterior, cambia totalmente la dinámica de producción del suelo urbano y marca la llegada de los actores de la especulación inmobiliaria. Por ejemplo, sobre el mismo eje Oeste, la "refundación" de Merlo en 1859, emprendida por el empresario y político Juan Dillon, estuvo enfocada al negocio inmobiliario que supuso la llegada del ferrocarril. 


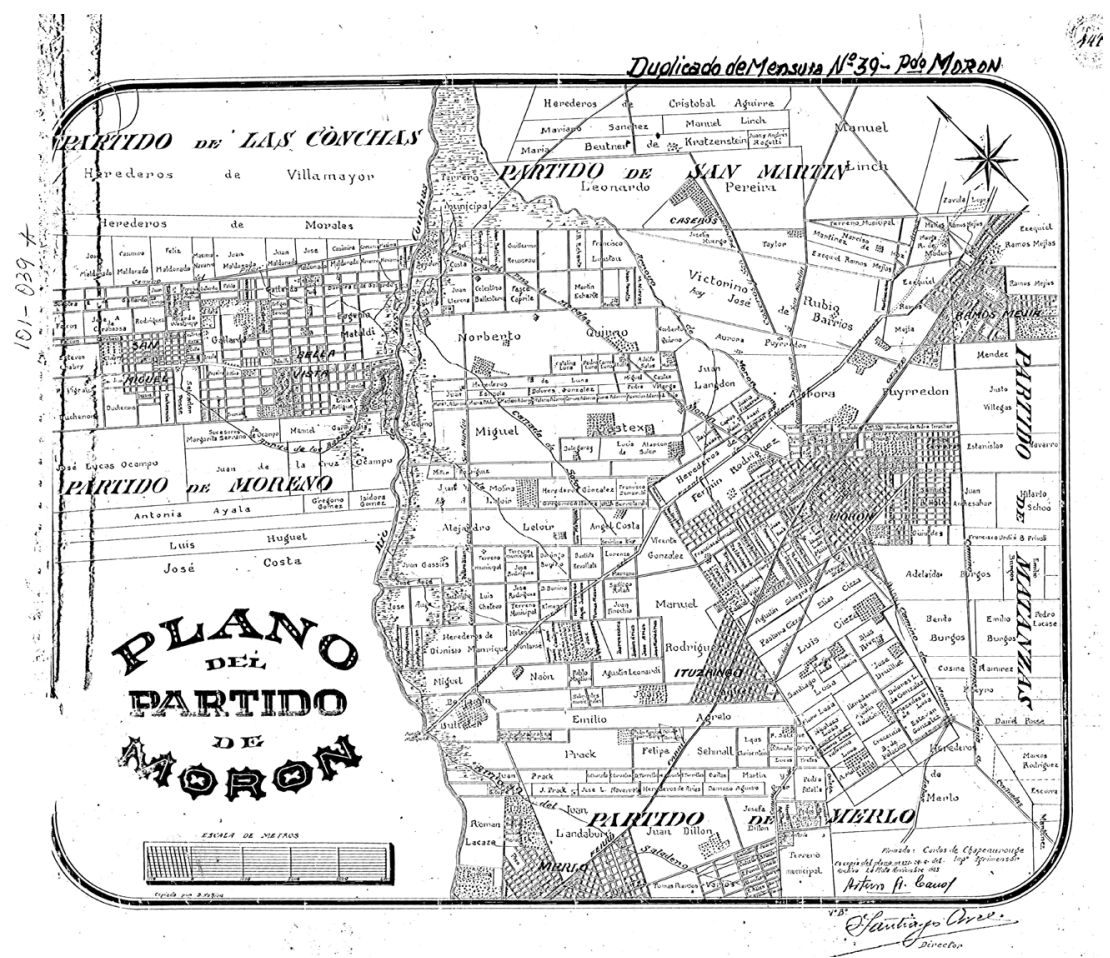

Figura 4 | Plano del partido de Morón, Mensura 39 del partido de Morón, Adolfo Soredeaux, 1866

FUENTE: DEPARTAMENTO DE INVESTIGACIÓN HISTÓRICA Y CARTOGRÁFICA (DIHC)

El pueblo de Ituzaingó, en el partido de Morón, (figura 4) fue creado por iniciativa de Manuel Rodríguez Fragio, un inmigrante gallego llegado al país en 1828. Rodríguez Fragio había comprado estas tierras en 1864 -seguramente consciente de su ubicación estratégica-, junto con otras importantes extensiones en los partidos de Merlo y Morón. Unos años más tarde, en 1872, negoció la apertura de una estación intermedia entre Merlo y Morón con el ferrocarril Oeste y pidió la aprobación al Gobierno respecto de la traza del pueblo de Santa Rosa de Ituzaingó. El servicio de trenes recién comenzó en 1884.

En el caso de Ramos Mejía, este núcleo nació cuando en 1858 se inauguró el apeadero. Los miembros de la familia Ramos Mejía, propietarios de los terrenos, donaron cuatro manzanas para edificios públicos, advirtiendo la importancia que podría cobrar esa parada en el corto plazo. Y efectivamente, con los años, los terrenos fueron cambiando de dueño por herencia o por venta hasta concretar la parcelación de las propiedades alrededor de la estación.

Haedo, ubicada sobre el mismo ramal ferroviario, surgió casi de la misma manera. Cuando se habilitó la estación en 1886, se realizó el loteo de las tierras a su alrededor. Los nuevos dueños pidieron al Gobierno la creación de un pueblo en este lugar, que fue aceptada en 1889 (figura 5). 


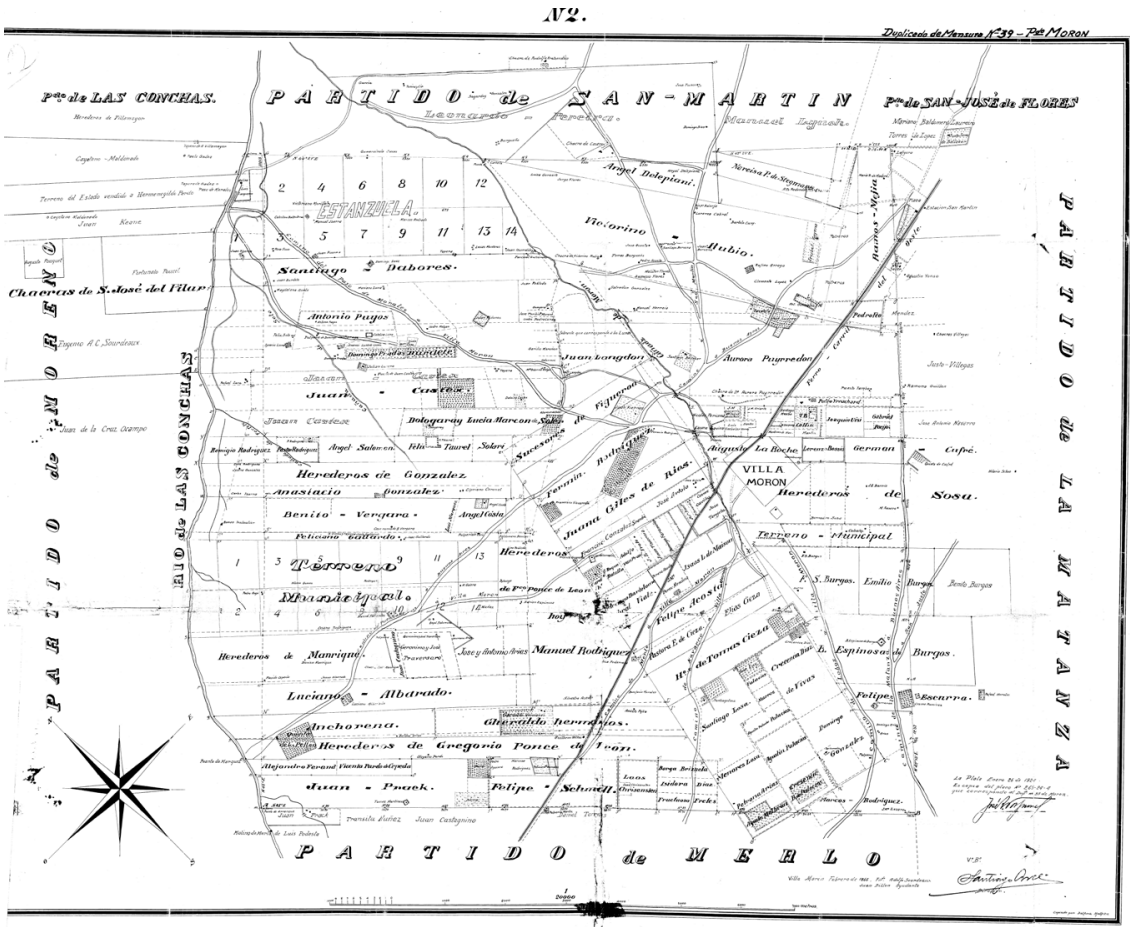

FIgura 5 | Plano del partido de Morón, copia del 1923 del Plano de Chapeaurouge de c. 1885

FUENTE: DEPARTAMENTO DE INVESTIGACIÓN HISTÓRICA Y CARTOGRÁFICA (DIHC)

De forma paralela a este proceso de establecimiento de pueblos, a finales del siglo XIX se produjo un cambio administrativo de gran importancia a partir de la federalización de la ciudad de Buenos Aires (1880), lo cual dio lugar a la reestructuración de la provincia de Buenos Aires y a la fundación de la ciudad de La Plata como su nueva capital. A nivel institucional, las repercusiones de estos cambios fueron notables. Desde su creación en 1875, el Departamento de Ingenieros de la provincia de Buenos Aires absorbió las tareas del DT, divididas y ampliadas en cuatro áreas principales: catastro, geodesia, puentes y caminos, y obras públicas. Una década más tarde, en 1885, fue creado el Ministerio de Obras Públicas de la Provincia (MOP), a raíz del aumento de los trabajos en el interior bonaerense y en la nueva capital provincial. El Departamento de Ingenieros pasó a ser una de las dependencias del MOP, hasta que se disolvió en 1913, cuando parte de sus funciones originales pasaron a la Sección de Geodesia y quedaron separadas de la obra pública (Fernández, 2014a, 2014b).

Esta progresiva división y especialización de funciones dentro del sector técnico estatal en lo concerniente al tema tierras tuvo amplias consecuencias en los procedimientos para aprobar los nuevos trazados de poblaciones y crecimiento de las mismas. Al mismo tiempo, los pedidos de trazados se multiplicaron debido al 
creciente valor que cobraba la tierra urbana en un contexto de urbanización tanto en el interior bonaerense como en el área de influencia de Buenos Aires (figura 6).

El siguiente periodo supondrá la aparición de los crecimientos entre los núcleos mencionados en este periodo a partir del auge de la especulación inmobiliaria, por el interés que cobra el suelo urbano. El crecimiento intersticial que se produce en la primera mitad del siglo xx es el que acaba por dar continuidad a la trama urbana desde Buenos Aires hasta Moreno.

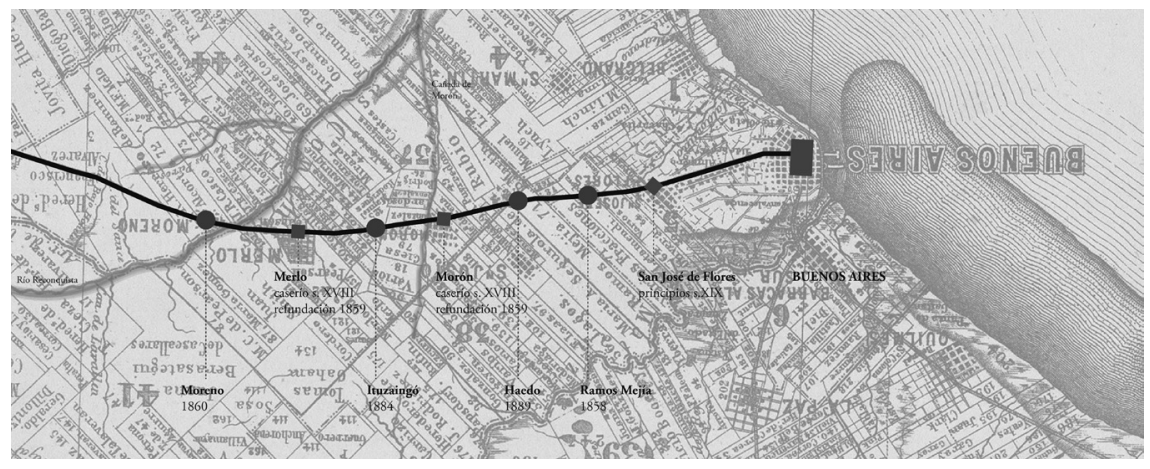

FIGURA 6 | Diagrama de la situación del corredor Oeste hacia 1890, que incluye los nuevos poblados establecidos sobre el trazado ferroviario

FUENTE: ELABORACIÓN PROPIA CON BASE EN REGISTRO GRÁFICO DE TIERRAS DE I 864

\section{Periodo 2: El crecimiento intersticial}

A partir de la década de 1910 , se asistió en el área metropolitana al crecimiento de un tipo particular de suburbio moderno, articulado en torno a nuevos loteos que se promocionaban para la residencia de verano o de fin de semana. Esta periferia se organizó por fuera de los límites de la ciudad, justo en el momento en que dichos límites se materializaban a partir de la construcción de la avenida de circunvalación General Paz (1936-1941). Conjuntamente con la consolidación de la capital, se hacía evidente un proceso de expansión que la sobrepasaba ampliamente y se extendía sobre los espacios intersticiales de los tres brazos principales de la urbanización (Norte, Oeste y Sur).

En las áreas más cercanas a la capital, comenzaron las ofertas de loteos económicos para trabajadores que se radicaban en las cercanías de las pequeñas industrias o talleres ubicados en el primer anillo de la expansión. En las áreas más alejadas, en un radio de unos $30 \mathrm{~km}$ desde el centro porteño, se fueron subdividiendo, primero, las grandes parcelas destinadas a quintas de fin de semana y a residencias secundarias de una clase media en pleno crecimiento, ubicadas generalmente sobre la franja de tierras a la vera de la línea ferroviaria. Un poco más tarde, durante los años treinta y cuarenta, los nuevos caminos permitieron el acceso a las tierras intersticiales, que hasta el momento permanecían sin subdividirse a causa de su difícil accesibilidad. Este proceso estuvo guiado mayormente por la acción privada: los dueños de la 
tierra, en conjunto con un agrimensor $-\mathrm{y}$, en algunos casos, de una empresa inmobiliaria-, subdividían las tierras que luego se vendían a plazos en remates públicos.

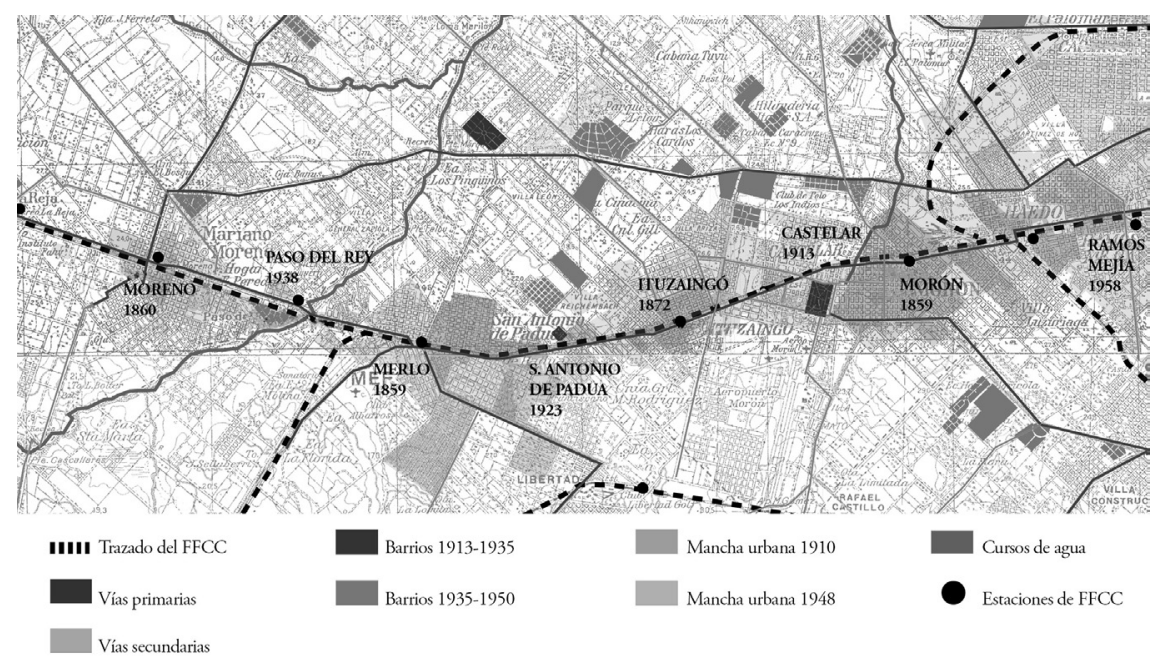

FIgURA 7 | Plano de la situación del eje Oeste hacia 1930, con los nuevos loteos de la época

FUENTE: ELABORACIÓN PROPIA CON BASE EN CARTOGRAFÍA DEL INSTITUTO GEOGRÁFICO MILITAR (IGM; HOY INSTITUTO GEOGRÁFICO NACIONAL, IGN)

Este dinamismo del mercado inmobiliario dio lugar a la aparición de un conjunto de compradores muy diverso interesado en la expansión. Algunos loteos generaron nuevos barrios residenciales que intentaron emular el suburbio jardín anglosajón; otras familias compraban un "terrenito" barato pensando en construir de a poco la casa de fin de semana; por último, otros compradores adquirían tierras en cuotas a modo de inversión, esperando el aumento de valor para una futura reventa. ${ }^{8}$

Los procesos que aquí mencionamos estaban regulados por la Ley 3487 de 1913, denominada de "Fundación de pueblos y ampliación de ejidos". Si bien la base de la ley puede rastrearse en la normativa del siglo XIX, las principales modificaciones tenían que ver con las trasformaciones en los cuerpos de gestión y control que mencionamos al final del apartado anterior. Desde el punto de vista político, la creación del Ministerio de Obras Públicas de la provincia forma parte de las medidas adoptadas por el nuevo Estado provincial, en el marco de un proceso de centralización de las decisiones a favor de su fortalecimiento. Sin embargo, como señalan Béjar (2002) y Cortabarría (2008), la evolución de la política de la provincia muestra que la subordinación de los municipios a las directivas del gobierno central condujo a una constante puja entre las partes: con periodos de marcado desequilibrio (18851910), de creciente autonomía municipal (1910-1930), hasta la intervención de muchos municipios durante el gobierno de facto iniciado en 1930 (figura 7).

8 Las ganancias previstas para las inmobiliarias y dueños que subdividían sus terrenos durante la primera mitad del siglo xx, podían llegar a triplicar los gastos de inversión. Véase Guaraglia (1971) y el folleto de la compañía Vinelli: “1906-2006. 100 años de Experiencia y Dedicación en el Mercado Inmobiliario". 
Ahora bien, ¿cuáles fueron las implicancias de estos cambios? La ley de 1913 establecía que la mensura y los planos del predio sujetos a enajenación, al igual que la división y subdivisión que se intentara dar al loteo, debían estar aprobados por los cuerpos técnicos del MOP. En efecto, estos eran los encargados de otorgar permisos a los propietarios de los terrenos destinados a la formación de los pueblos y también de elaborar los informes técnicos (trazados, condiciones topográficas y desagües de la tierra), que luego serían aprobados - o no- por el Poder Ejecutivo. En la práctica, y por la falta de capacidad del organismo central, en reiteradas ocasiones se solicitaba a los cuerpos municipales que verificaran los trazados in situ, y elevaran luego un informe a las oficinas centrales-Geodesia del MOP- (Gómez Pintus, 2015). De este modo, gran parte de las aprobaciones, de los informes y, más en general, de los canales de gestión de los mismos, eran permeables a las relaciones políticopersonales que se jugaban entre intendentes municipales y el gobierno provincial.

Por otro lado, retomando la ley de 1913, se verifica que "la ampliación de ejidos" había constituido una preocupación en cierta forma secundaria y, por lo tanto, se reconocía un mayor grado de indeterminación legal. Sin embrago, por el tipo de expansión fragmentaria que tuvo lugar en los alrededores de Buenos Aires, dicho proceso terminó afectando a la mayor parte de los loteos, ya que varios de ellos no conformaron nuevos centros de población, sino que quedaron enmarcados bajo el concepto de "ampliación del ejido" (dentro de la zona de quintas o chacras). De manera implícita, podría entenderse que la nueva reglamentación "alentaba" la expansión sobre la zona de quintas en los alrededores de los pueblos existentes, con una forma y una reglamentación más laxa. Concretamente, esto implicó, entre otras cosas, la posibilidad de eximirse de ceder tierras para reserva urbana, provocando que muchos de estos emprendimientos carecieran de solares destinados a equipamientos públicos o bien a zonas verdes, dado que en el artículo 5 de la mencionada ley se planteaba que "en las zonas de ampliación, el Poder Ejecutivo podrá eximir al propietario de la obligación de dejar alguna o algunas de las reservas indicadas en el Artículo 14 de esta ley".

Sobre la base de este debate, proponemos el acercamiento a un caso concreto en Ituzaingó para verificar los "huecos" que dejaba a la vista la aplicación de la normativa. Los trámites realizados ante la Dirección de Geodesia del mop para la aprobación del trazado del Barrio del Golf Ituzaingó (San Antonio de Padua, 1931) nos proveen información detallada para ilustrar esta situación. En el año 1931, el ingeniero Armando Frhener, autorizado por la Compañía de Tierras del Oeste, solicitaba al director de Geodesia y Catastro la aprobación del plano del "Barrio del Golf" (figura 8):

Se trata de regularizar hechos existentes, ya que varios propietarios han comprado lotes, edificado y plantado árboles de buena fe y a fin de no dañar sus intereses y allanar en lo posible las dificultades de la Compañía, por cuya razón pido se contemple lo relacionado con el trazado de las calles que no tienen el ancho reglamentario. Los lotes del nuevo proyecto tienen todos frente mínimo de $10 \mathrm{mts}$ con excepción de unos pocos que no se pudieron modificar por estar vendidos [...]. 
Confío que el Sr. Director quiera considerar la necesidad de regularizar la actual situación en el sentido de allanar en lo posible las dificultades y contribuir a la buena fe de la Compañía de Tierras con una resolución favorable que venga a sanear un estado de cosas que puede ser causa de confusiones y pleitos [...].

Se considera también que no le corresponde a la Compañia de Tierras la cesión de Reservas para uso Público considerando la pequeña cantidad de terreno propiedad de la misma y el hecho de que el pueblo de San Antonio ya posee escuela, iglesia y comisaría en las inmediaciones de los terrenos en cuestión. Además cuando la Municipalidad aprobó los planos presentados por el Sr. Benavides no obligó a Diaz Valdéz (a la sazón propietario de las tierras) a ceder las reservas antes mencionadas. (Solicitud de aprobación del trazado del "Barrio del Golf", en Copia de Mensura, Duplicado no 54, Merlo, ańo 1931. Departamento de Investigación Histórica y Cartográfica [DIHC]. Destacados nuestros)

La aprobación definitiva del barrio llegó en 1936, cuando se aceptó el plano presentado pese al incumplimiento en relación con las medidas de algunos lotes y calles, y se exigió escriturar a favor del fisco una parte de las reservas correspondientes.

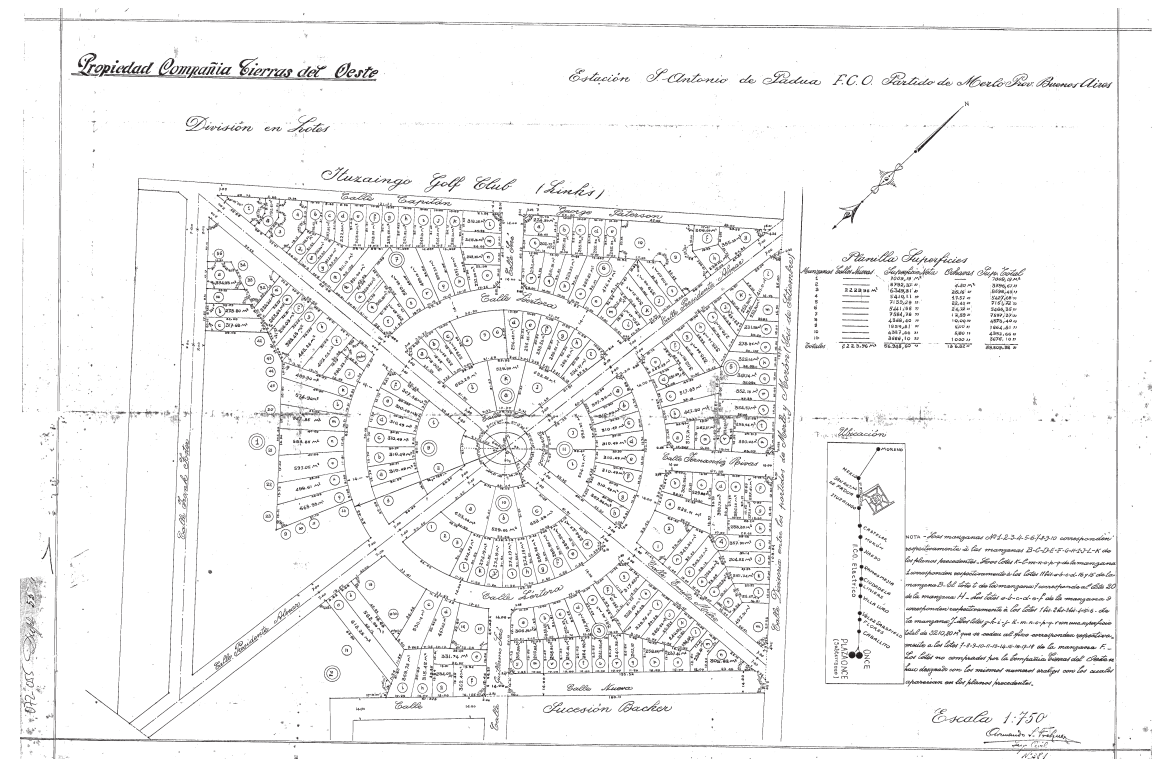

Figura 8 | Plano del Barrio del Golf, en la Estación Sn Antonio de Padua FUENTE: DEPARTAMENTO DE INVESTIGACIÓN HISTÓRICA Y CARTOGRÁFICA [DIHC]

La resolución final del caso muestra la voluntad de hacer respetar ciertas normas, aunque también permite esgrimir que algunas "licencias o excepciones", como la medida de los lotes o el porcentaje de reservas que se cedían al Municipio, podían ser admitidas como prácticas cotidianas. En este sentido, si tensionamos al máximo esta posibilidad de exención, podemos imaginar como resultado la materialización de áreas suburbanas continuas, conformadas a partir de la sumatoria de sucesivos loteos de pequeñas dimensiones, carentes completamente de reservas para el uso público. 
Este escenario se hace presente en amplios sectores del Gran Buenos Aires, como lo demuestran los primeros relevamientos catastrales del área en 1948, o como denunciaron incansablemente los representantes municipales ante la Comisión Asesora del Gran Buenos Aires (Gómez Pintus \& Fernández, 2016).

\section{Conclusiones}

El estudio de la interacción entre los diferentes actores que producen el suelo urbano a lo largo de más de cien años, nos demuestra que, pese a la presencia de un cuerpo legislativo que regulaba específicamente cómo debían ser los nuevos asentamientos, la situación distaba mucho de ser una imposición "desde arriba" de un modelo de ciudad. La producción de un nuevo asentamiento urbano y sus extensiones suponía, por lo tanto, una arena de conflictos y negociaciones entre los organismos técnicos (encargados de aplicar las normativas) y los actores locales (que buscaban los intereses propios o comunes del municipio referidos a un negocio inmobiliario más próspero o una mejor calidad urbana).

Analizar cómo se realizaron determinados trazados nos brinda la oportunidad de observar cuáles eran los temas de conflicto a nivel de los pueblos, cómo se negociaban, quiénes eran los actores involucrados y cómo se consensuaba el resultado que, finalmente, el organismo de gobierno central debía aprobar (tabla 1).
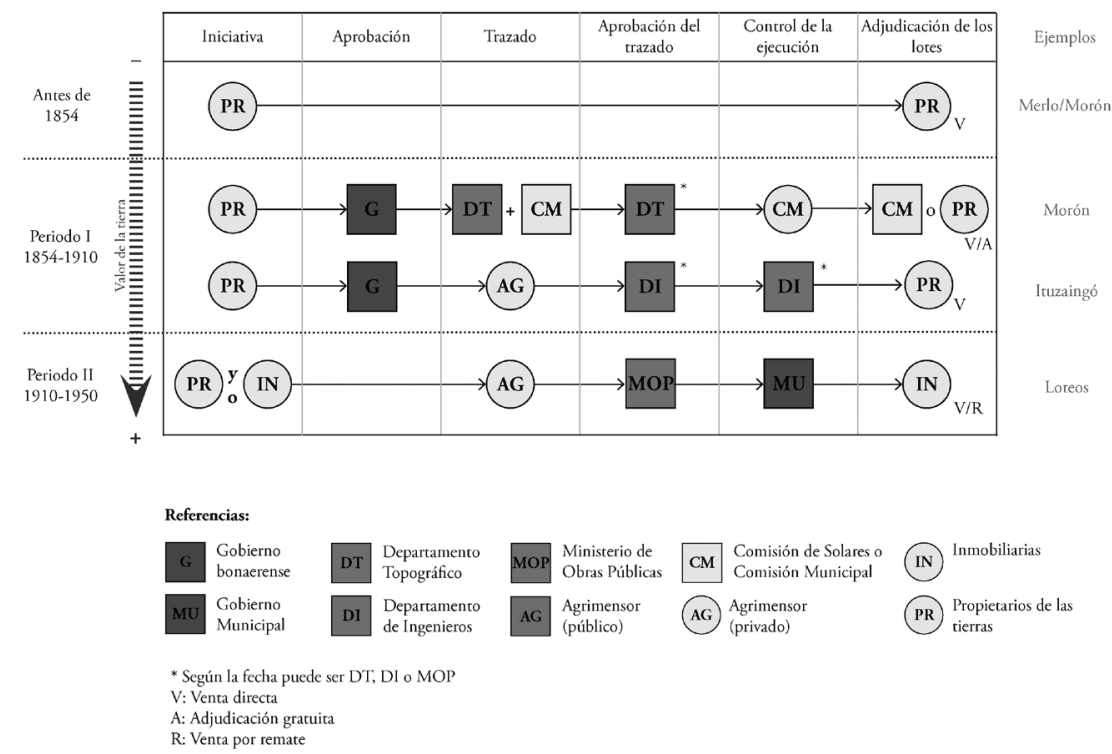

TABLA I | Proceso de producción del suelo urbano en el Eje Oeste en los periodos analizados

FUENTE: ELABORACIÓN PROPIA 
Antes de la llegada del ferrocarril, identificamos el germen de los poblados en las iniciativas de los propietarios que dieron lugar al establecimiento de capillas y/o postas alrededor de las cuales se concentraron población y actividades diversas. El pueblo creció gracias a la donación de tierras o al permiso del propietario para establecer un asentamiento en ellas, lo cual también redundaba en su propio beneficio.

La "regularización" de estos poblados, sin embargo, no fue posible hasta la década de 1850. A partir de este momento, las relaciones se fueron complejizando en las tierras próximas a la ciudad de Buenos Aires, y ello por dos motivos principales. En primer lugar, la llegada del ferrocarril implicó un cambio rotundo en el valor del suelo, debido a la conexión rápida que supuso entre la capital nacional y los pueblos de alrededor. En segundo lugar, por la voluntad (y la necesidad) de "regularizar" la trama urbana de los poblados existentes, asociada a la reorganización del Departamento Topográfico tras el ocaso de la etapa rosista.

En este primer periodo, la acción de las Comisiones de Solares -más tarde llamadas Comisiones Municipales, y que ya existían desde mucho antes-, se tornó central para comprender las negociaciones entre el Estado provincial en construcción y los poderes locales. Las formas de tenencia de la tierra y de los espacios ocupados debían normalizarse, para poder convertirlos en un objeto con alto valor de intercambio. Estamos ante el nacimiento del mercado inmobiliario en los alrededores de Buenos Aires. Aquí, el Estado provincial, a través de la figura de los agrimensores, negociaba con el pueblo, representado por su Comisión Municipal, la forma que tendría la ciudad. No se trata de ninguna manera de la imposición de un modelo legal sobre el territorio, sino de una instancia de negociación en la que se iban determinando los anchos de calles, la ubicación de las plazas, la forma de dirimir los conflictos con los propietarios afectados y las posibles resoluciones... todo ello sin olvidar que el resultado debía ser una forma urbana lo más "regular" posible. Este consenso evitaba futuros pleitos entre los propietarios, que era algo que el Departamento Topográfico se esforzaba por lograr, tanto en las tierras rurales como en las ejidales.

En el segundo periodo, ya en el siglo xx, la dinámica volvió a cambiar. Es notable el aumento de las operaciones de subdivisión y venta de tierras, pues comenzaron a rellenarse los intersticios entre los pueblos o estaciones ferroviarias y, al mismo tiempo, entró el transporte automotor en escena, en detrimento del ferrocarril.

El problema central que identificamos en este periodo es el de definir qué tipo de suelo urbano se estaba produciendo: nuevo pueblo o extensión del mismo. Frente a tal alternativa, los actores se esforzaban en encontrar los "agujeros legales" en la normativa que les permitieran generar crecimientos suburbanos modernos con una ley decimonónica.

El sistemático pedido de excepciones a la norma imperante en el momento de aprobar los nuevos crecimientos estaba fundamentado en que se estaban construyendo "ensanches de ejido" y no "nuevas poblaciones". Esto eximía a los promotores de reservar terrenos para usos públicos y/o equipamientos, que a la larga redundarían en crecimiento fragmentarios sin centralidades claras ni espacios de referencia -solo en 1968 la ley contemplaría la obligatoriedad de la instalación de servicios básicos antes de aprobar el loteo-. 
Por otra parte, el análisis efectuado sobre la escala local de las decisiones urbanas nos permite verificar de modo empírico ciertas ideas propuestas desde las ciencias sociales, las cuales promueven que la historia de la formación del Estado argentino es también un proceso lento de centralización.

A lo largo del recorrido analizado, observamos que la Municipalidad va perdiendo injerencia sobre la forma del trazado. En una primera etapa, durante casi todo el siglo XIX, se produce un consenso entre la Municipalidad (representada por la Comisión de Solares o la Comisión Municipal) y el organismo técnico, que da como producto un trazado consensuado. Sin embargo, ya desde principios del siglo xx esta relación cambia, esencialmente por la ampliación en el número de actores y porque se promueve una relación entre el loteador, dueño de la tierra, y el organismo técnico estatal que, al menos desde la letra de la ley, deja fuera de juego a la Municipalidad.

La creación del Ministerio de Obras Públicas en 1885 y la ley de 1913, contribuyen a acentuar esta pérdida de poder de decisión de la escala municipal, dado que se da a la Dirección de Geodesia la potestad de aceptar o no los trazados presentados por los privados, aunque -como mencionamos- en la práctica siguen siendo los técnicos municipales los que verifican sobre el territorio. Este objetivo de centralización, independientemente de la lentitud con la que se haya logrado, adquiere sentido si tenemos en cuenta que comienzan a delimitarse funciones acordes a la "nueva provincia" creada a partir de la nacionalización de Buenos Aires en 1880, pues se trata de reconstruir una provincia capaz de ejercer legitimidad (física y simbólica) en todo el territorio.

Con este análisis se demuestra que, a lo largo del periodo estudiado, la normativa se mantiene relativamente estática, mientras que es la interacción entre los actores productores del suelo urbano lo que va cambiando. En el primer periodo, el consenso se produce in situ; es decir, existe un encuentro real entre los actores que deciden cómo ha de ser la ciudad. En el segundo periodo, la centralización de los trámites y aprobaciones en el Ministerio de Obras Públicas de la Provincia, los nuevos canales, junto a la creciente burocratización, despersonaliza el seguimiento de las aprobaciones, de los pedidos de excepción y especialmente de su control. Esta misma lejanía entre los actores que deciden el trazado y quienes lo aprueban, y ciertos "agujeros legales", dan lugar a la informalidad en algunos procesos -como, por ejemplo, la venta de tierras sin su aprobación previa-, y a la imposibilidad de verificar los trazados in situ desde los organismos técnicos.

Finalmente, verificamos que la supuesta "ausencia del Estado" no es tal. Si bien el Estado en el periodo analizado no planifica ni la localización de los nuevos pueblos ni los trazados de extensión o su ubicación, interviene de otras maneras que tienen que ver con la determinación de los procesos y la normativa que debe seguir la forma de la ciudad.

\section{Agradecimientos}

Este texto forma parte de una investigación iniciada en 2018, financiada por la Universidad Nacional de La Plata, PPID/Uoo8 "Construyendo la periferia: las formas de crecimiento de la Región Buenos Aires en la primera mitad del siglo xx y su representación cartográfica”. 


\section{Referencias bibliográficas}

Aliata, F. (2010). Transformaciones en el hábitat rural. Los planos topográficos de Chascomús, 1826-1854. Mundo Agrario, 10(20),1-35. http://www.memoria.fahce.unlp.edu.ar/ art_revistas/pr.4175/pr.4175.pdf

Aliata, F. \& Loyola, O. (2005). La acción del Departamento Topográfico y las Comisiones de Solares en la consolidación de los poblados bonaerenses. Dolores entre 1821 y 1838. En G. Batticuore, K. Gallo, \& J. Myers, Resonancias románticas. Ensayos sobre historia de la cultura argentina (1820-1890) (pp. 245-268). Buenos Aires: Eudeba.

Béjar, D. (2002). Los conservadores bonaerenses: un partido político desde el gobierno. Estudios Sociales. Revista universitaria semestral, 22-23, 95-122.

Birocco, C. M. (2009). Del Morón rural al Morón urbano. Vecindad, poder y surgimiento del Estado Municipal entre 1770 y 1895. Buenos Aires: El autor.

Bohoslavsky, E. \& Soprano, G. (2010). Un estado con rostro humano. Funcionarios e instituciones estatales en Argentina (desde 1880 a la actualidad). Buenos Aires: Universidad Nacional de General Sarmiento (UnGs) / Prometeo.

Canedo, M. (2011). Mucho más que una cuestión de medidas. Las comisiones para el arreglo de los pueblos del Estado de Buenos Aires: Pergamino, Arrecifes, San Pedro, 18541856. En J. C. Garavaglia \& P. Gautreau, Mensurar la tierra, controlar el territorio: América Latina, siglos XVIII-XIX (pp. 259-293). Rosario: Prohistoria.

Canedo, M. (2013). Cartas entre agrimensores. Miradas desde los pueblos en un periodo de transiciones políticas, institucionales y sociales (Estado de Buenos Aires, 1854-1856). Revista Electrónica de Fuentes y Archivos. Centro de Estudios Históricos "Prof. Carlos S. A. Segreti”, 4(4), 209-222. https://refa.org.ar/file.php?tipo=Contenido\&id=102

Canedo, M. (2014). El 'restablecimiento' del Departamento Topográfico de Buenos Aires. Política y gestión de un proyecto con consenso (1852-1857). Andes, 25(2). http://bdt. unsa.edu.ar/ojs/index.php/Andes/article/view/183/170

Clichevsky, N. (1975). El mercado de tierras en el área de la expansión de Buenos Aires y su incidencia sobre los sectores populares, periodo 1943-1973. Buenos Aires: Centro de Estudios Urbanos y Regionales, Instituto Torcuato Di Tella.

Clichevsky, N. (2002). Tierra vacante en ciudades latinoamericanas. Toronto: Nora Clichevsky Editora.

Cortabarría, J. J. (2008). El régimen municipal bonaerense de 1891-1955. Revista Electrónica del Instituto de Investigación "Ambrosio L. Gioja", 2(3), 8-31. https://dialnet.unirioja.es/ servlet/articulo? codigo $=4357517$

Cortés Conde, R. (1979). El progreso Argentino: 1880-1914. Buenos Aires: Sudamericana.

Cricelli, S. (2008). La política territorial y la estructura de la Administración en relación con los pueblos de campańa en el periodo posrevolucionario: Organismos centrales y locales. Revista Registros, 5, 51-61.

Cricelli, S. \& Galcerán, V. (2013). La gestión del territorio en la provincia de Bs. As., sus instituciones y actores (1821-1854). XIV Jornadas Interescuelas/Departamentos de Historia. Mendoza: Departamento de Historia de la Facultad de Filosofía y Letras, Universidad Nacional de Cuyo. http://cdsa.aacademica.org/000-010/609.pdf 
D’Agostino, V. (2015). Estado, instituciones y funcionarios bonaerenses: la reorganización de la repartición topográfica en la segunda mitad del siglo xix. En M. Blanco \& L. Barandiarán (comps.), Las configuraciones de la trama social. Políticas públicas, instituciones y actores en la Argentina contemporánea. Tandil: Universidad Nacional del Centro de la Provincia de Buenos Aires (Unicen).

Favelukes, G., Novick, A., \& Zanzottera, G. (2016). Cartografías del Área Metropolitana de Buenos Aires según Patricio Randle, César Vapńarsky y Horacio Torres. Estudios del Hábitat, 14(2) e010. https://revistas.unlp.edu.ar/Habitat/article/view/e010/pdf

Favelukes, G. \& Novick, A. (eds.) (2017) Anales del Instituto de Arte Americano, Bordes Metropolitanos, 47(2). http://www.iaa.fadu.uba.ar/ojs/index.php/anales/issue/view/47 $\% 282 \% 29-2017$

Fernández, N. (2014a). Estado, política y obras públicas. El Ministerio de Obras Públicas de la Provincia de Buenos Aires, 1917-1943. IV Jornada de Becarios y Tesistas. Bernal, Universidad Nacional de Quilmes. http://sociales.unq.edu.ar/wp-content/uploads/ byt2014/ponencias/eje06/FernandezNoelia-E stadopoliticayobraspublicas.pdf

Fernández, N. (2014b). Estado, administración y procesos políticos. El Ministerio de Obras Públicas de la Provincia de Buenos Aires entre 1917 y 1943. VII Jornadas de Sociología de la UNLP. Ensenada, Facultad de Humanidades y Ciencias de la Educación, Universidad Nacional de La Plata. http://jornadassociologia.fahce.unlp.edu.ar/viii-jornadas-2014/ PONmesa18FernandezN.pdf

Garavaglia, J. C. \& Gautreau, P. (2011). Mensurar la tierra, controlar el territorio: América Latina, siglos XVIII-XIX. Rosario, Argentina: Prohistoria.

Gómez Pintus, A. (2015). Barrios parque y de fin de semana en el mapa de la expansión metropolitana de Buenos Aires. 1910-1950. EURE, 41(123), 159-185. http://dx.doi. org/10.4067/S0250-71612015000300007

Gómez Pintus, A. \& Fernández, N. (2016). La formación del Gran Buenos Aires (Argentina) a través del análisis de las normativas y códigos vigentes (1910-1927). URBE Revista Brasileira de Gestão Urbana, 8(2), 242-260. http://dx.doi.org/10.1590/21753369.008.002.AO06

Gorelik, A. (2015). Terra Incognita. Para una comprensión del Gran Buenos Aires como Gran Buenos Aires. En G. Kessler (dir.), El Gran Buenos Aires. Colección Historia de la Provincia de Buenos Aires, tomo 6 (pp. 21-69). Buenos Aires: unipe-edHASA.

Guaraglia, L. (1971). Manual del rematador. Buenos Aires: Ediciones Machi.

Heras, C. (1949). Antecedentes sobre la instalación de las municipalidades en la Provincia de Buenos Aires (1852-1854). Trabajos y Comunicaciones, 1, 75-89.

Jaramillo, S. (1981). Producción de vivienda y Capitalismo dependiente: el caso de Bogotá. Bogotá: Dintel.

Lovera, A. (2012). El capital inmobiliario y constructor en la producción de la ciudad en América Latina. En B. Ramírez Velázquez \& E. Pradilla Cobos (comps.), Teorías sobre la ciudad en América Latina I (pp. 319-370). México, DF: Universidad Autónoma Metropolitana.

Loyola, O. \& Zweifel, T. (2013). La acción del Departamento Topográfico y las Comisiones de Solares en la consolidación de los poblados bonaerenses. El partido de San José de Flores. 1829-1836. Estudios del Hábitat, 11,38-45. http://sedici.unlp.edu.ar/bitstream/ handle/10915/33032/Documento_completo.pdf?sequence=1\&isAllowed=y 
Mulhall, M. (1875). Handbook of the river Plate republics. Comprising Buenos Ayres and the provinces of the Argentine Republic and the republics of Uruguay and Paraguay. Londres: Edward Stanford Ltd.

Oszlak, O. (1982). La formación del Estado argentino: orden, progreso y organización nacional. Buenos Aires: Planeta.

Pesoa, M. (2016). Una ciudad para la pampa: la construcción del territorio en la Provincia de Buenos Aires (1810-1916). Tesis doctoral inédita, Departamento de Urbanismo y Ordenación del Territorio, Universitat Politècnica de Catalunya. https://upcommons. upc.edu/handle/2117/96228?locale-attribute=es

Sabatini, F. \& Arenas, F. (2000). Entre el Estado y el mercado: resonancias geográficas y sustentabilidad social en Santiago de Chile. EURE, 26(79), 95-113. http://dx.doi. org/10.4067/S0250-71612000007900006

Sáez, G. (dir.) (2014). Sintesis histórica del partido de Morón. Haedo: Instituto y Archivo Histórico Municipal de Morón.

Sargent, Ch. (1974). The spatial evolution of Greater Buenos Aires 1870-1930. Tempe, Az: Arizona State University, Center for Latin American Studies.

Schteingart, M. (1989). Los productores del espacio habitable: Estado, Empresa y sociedad en Ciudad de México. México DF: El Colegio de México.

Schwarzer, J. \& Gómez, T. (2006). La primera gran empresa de los argentinos. El ferrocarril Oeste (1854, 1962). Buenos Aires: Fondo de Cultura Económica.

Smolka, M. (1981). Precio de la tierra y valorización inmobiliaria urbana: esbozo para una conceptualización del problema. Revista Interamericana de Planificación, 15(60), 7089.

Smolka, M. (1987). O capital incorporador e seus movimentos de valorizacáo. Cadernos IPPUR/ UFRJ, 2(1), 41-78.

Topalov, Ch. (1987). Le Logement en France. Histoire d'une marchandise impossible. París: Presses de la Fondation Nationale des Ciences Politiques.

Vapñarsky, C. (2000). La aglomeración Gran Buenos Aires. Expansión espacial y crecimiento demográfico entre 1869 y 1991. Buenos Aires: EudeBA. 